\title{
Migración en Chile, una experiencia desde el proceso individual de la resiliencia
}

\author{
Migration in Chile: an experience from the individual \\ process of resilience
}

Ana Castro Ríos

Eugenio Saavedra Guajardo

Universidad Católica del Maule

\section{Resumen}

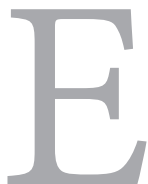

1 capítulo que se presenta a continuación, da cuenta de un estudio cualitativo, desde la perspectiva de la resiliencia, sobre el fenómeno de migración que se está produciendo en Chile; situación social que actualmente está configurando cambios importantes en la sociedad chilena. Se evidencia la experiencia individual de un sujeto, que desde los núcleos de significados asociados a riesgo, protección y conductas resilientes, describe como el proceso de migración lo ha transformado en una oportunidad de salir adelante y configura su presente. La migración refleja, hoy especialmente en nuestra América Latina, procesos de pobreza y desigualdad importantes y sostenidos, que el modelo económico imperante y el rol asumido por el Estado, agudizan implacablemente.

\section{Palabras claves:}

Migración, desigualdad, resiliencia, resignificación.

\section{Summary}

$\mathrm{n}$ the chapter that we are to present, we inform about the qualitative study, from the perspective of resilience, on the migration phenomenon that is happening in Chile; social situation in which important changes are taking place currently in the Chilean society. It is noticeable the individual experience of a person, who from his personal perception of meaning regarding risk, protection and resilient behavior, describe how this process of migration has transformed it into an opportunity to be successful and keep going forward to shape his present. Migration represents, especially today in Latin America, the processes of poverty and important marked inequality, which are determined inexorably by the current economical model and the role adopted by the government, which are making it worse.

\section{Key words:}

Migration, inequality, resilience, resignification. 


\section{Ciencia}

\section{Introducción}

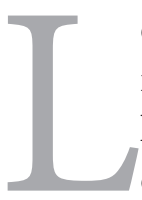

os procesos de migración, indicados como inmigración y emigración para destacar la entrada o salida a un país como dirección del movimiento, es parte del proceso de poblamiento de la sociedad. Históricamente se han desarrollado movimientos migratorios, que han ido dando forma a los actuales países, la conformación de sus territorios y sus culturas.

Los estudiosos del tema, indican que se han ido desarrollando patrones en torno a este fenómeno y señalan que en cuanto al proceso en América, fundamentalmente entre la segunda mitad del siglo XIX y la primera del XX, se produce la inmigración denominada de ultramar (Villa \& Martínez, 2001). Esta tuvo su origen principalmente desde el sur de Europa y tuvo incidencia en la configuración de varias sociedades nacionales de nuestra América.

Esta inmigración se relacionó de manera importante con los circuitos económicos internacionales y les permitió de manera sostenida crear polos de desarrollo. Sin embargo, a contar de los años 70 y en virtud de la escasa renovación de los flujos comerciales, estos grupos de inmigrantes, comienzan a mostrar un sostenido envejecimiento; "en este contexto, la mortalidad y la migración de retorno redundaron en una gradual merma de su stock, cuya cifra estuvo bajo los 2.5 millones de personas hacia 1990" (Villa, 2001, p.13).

El patrón migratorio denominado extra- regional, ha ido adquiriendo un rol protagónico en las últimas décadas. Este se caracteriza por encontrarse, mayoritariamente en los Estados Unidos y, en menor cantidad en Canadá y se le ha denominado el corredor norte-sur. Este patrón de migración es el más importante en la Región y ha tenido múltiples repercusiones para los países del norte de América Latina, especialmente México y para el Caribe; entre las más importantes, según los mismos autores, se destacan la pérdida de recursos humanos calificados y la exposición de los emigrantes al riesgo de no lograr una efectiva inserción en los lugares de destino.

Esta migración también ha implicado la formación de comunidades transnacionales de migrantes y la generación de un potencial económico asociado a las remesas que los emigrantes envían a sus lugares de origen, tema que revisaremos más adelante.

Según Bajraj, Villa y Rodríguez (2000), la descripción de los patrones migratorios quedaría trunca si no se hace mención a los efectos más generales de la migración. Según sostienen,

\begin{abstract}
...en muchos países de la región la emigración ha contribuido a aliviar el impacto tanto de las tensiones entre las tendencias demográficas y la generación de empleo como de aquellas originadas en conflictos sociopolíticos, étnicos y religiosos o de las asociadas con formas agudas de degradación ambiental (p.36).
\end{abstract}

Asimismo, estos autores sostienen que la emigración se ha constituido en una alternativa para la búsqueda individual, fuera de los países de ori- 


\section{Ciencia}

gen, de mejores oportunidades laborales y de formación personal.

En relación a los países de inmigración, estos han enfrentado algunos problemas como la indocumentación y el surgimiento de percepciones negativas frente a los costos que involucra la utilización que hacen los inmigrantes de servicios sociales subsidiados (salud, educación, seguridad social). A pesar de ello, los países receptores han obtenido importantes beneficios de la inmigración, particularmente en cuanto al aprovechamiento de una oferta de mano de obra barata o altamente calificada, según sea el caso.

Retomando el tema de las remesas, Stefoni (2012) señala que el interés en estas es relativamente reciente y las razones que lo explican se vinculan al menos con cuatro factores: a) el aumento en el número de emigrantes, como ya se ha descrito, con lo que se establece algo evidente, a mayor número de emigrantes hacia economías desarrolladas, mayor cantidad de remesas ingresan a las familias, economías locales, regionales y nacionales de los países de origen. b) la visibilidad que se logra a partir de la cuantificación del monto que reciben los distintos países receptores y la constatación de que este número se ha incrementado en las últimas décadas. c) el hecho de que parte importante de los países receptores de remesas son aquellos que tienen mayores índices de pobreza, desigualdad y subdesarrollo, lo que lleva a ver en las remesas un factor clave en el desarrollo económico latinoamericano y d) un cuarto factor relacionado con la orientación que toma la política económica a nivel regional en un contexto de globalización de un modelo liberal de economía. En este escenario, el discurso respecto de que los migrantes pueden y deben jugar un rol fundamental en el desarrollo de sus comunidades, se visualiza como un discurso político que tiende a reafirmar un modelo de desarrollo donde el Estado termina ocupando una función de coordinación y apoyo, más que de gestor e impulsor del desarrollo.

Pareciera que, por lo menos a corto plazo, la migración tenderá a seguir aumentando, puesto que no se advierten aún indicios sólidos de una disminución de las grandes desigualdades en el grado de desarrollo relativo de los países.

Las relaciones entre la migración y la pobreza son complejas. Las evidencias indican que los grupos más pobres de la sociedad suelen ver restringida la posibilidad de mudarse de lugar de residencia, limitación que puede convertirse en una forma de confinamiento territorial; en otros casos, la migración de los pobres no parece obedecer al libre ejercicio de una opción sino más bien a los efectos de una virtual expulsión desde los lugares de origen (Bajraj et al, 2000).

Y especialmente en Chile, uno de los países con mayor índice de desigualdad (ocupa el $4^{\circ}$ lugar entre 34 países, según informe de la OCDE del año 2014), la migración es una realidad que recién comienza a perfilarse y por tanto a estu- 


\section{Ciencia}

diarse como tema, el cual es necesario hacerse cargo en investigaciones y políticas públicas.

\section{La micro experiencia personal de la mi- gración: una mirada desde la resiliencia.}

A partir de la afirmación de los teóricos de la modernización, según la cual los migrantes del campo a la ciudad debían ser los individuos con mentalidad más moderna dentro de sus comunidades de origen, se ha considerado que algunos rasgos de personalidad y ciertas actitudes hacia el cambio o los riesgos y la incertidumbre que esto implica, se relacionan con la decisión de migrar (Rodríguez \& Busso, 2009).

Sin duda son muchas opciones teóricas las que se pueden hacer cargo de esta inmersión del tema de la migración, desde la vivencia particular de un sujeto. Optamos en este estudio por lo que nos aporta la perspectiva de la resiliencia y para ello nos centraremos en los relatos de los sujetos en torno a su experiencia de riesgo, protección y conductas resilientes.

La palabra resiliencia tiene su origen en el latín, básicamente en el término "Resilio" que significa volver atrás, rebotar. Claramente el término fue adaptado a las ciencias sociales para caracterizar a los sujetos que a pesar de haber nacido y vivir en situaciones de adversidad, se desarrollan psicológicamente sanos y exitosos (Rutter, 1994 en Saavedra, 2011).

La resiliencia en este sentido, puede transformar o fortalecer a aquellos enfrentados a la ad- versidad, manteniendo conductas de adaptación, permitiendo un desarrollo normal o promoviendo un crecimiento más allá del nivel de funcionamiento presente. Lo anterior refuerza la idea de que un sujeto no solo puede sobreponerse a la adversidad, sino que es capaz de construir sobre ella, convirtiendo estos obstáculos en oportunidades de desarrollo.

Cuando hablamos de estar en riesgo, estamos aludiendo específicamente a un número de factores adversos para el sujeto. La pobreza, las desavenencias familiares, la violencia, el abuso de sustancias y las enfermedades están entre los factores de riesgo más nombrados (Saavedra, 2011).

Por otra parte, los denominados factores protectores, como a) presentar un acercamiento activo hacia el problema; b) habilidad para captar la atención positiva de los otros; c) desarrollar una visión optimista de sus experiencias; d) habilidad para mantener una visión positiva de una vida significativa; e) habilidad para estar alerta y mantener la autonomía; f) tendencia a buscar nuevas experiencias; y g) desarrollar una perspectiva proactiva (Saavedra, 2011).

Ademásdelosfactoresprotectoresantesmencionados, el autoconcepto construido juega un papel fundamental en la conducta resiliente.

De esta forma, la manera de entenderse a sí mismo y a los otros, establece los límites de comprensión para evaluar las situaciones vividas, dando significado y coherencia a las experiencias 


\section{Ciencia}

acumuladas por el sujeto (Guidano, 1994, en Saavedra, 2011).

La investigación que se presenta, pretende evidenciar a través de los discursos de los sujetos, de que manera elementos como: a) la autoestima, b) los vínculos afectivos y habilidades sociales, c) la creatividad y el humor, d) la red social y el sentido de pertenencia y e) el sentido de trascendencia; se encuentran presentes en la forma de enfrentar la experiencia de la emigración desde Colombia a Chile.

\section{Metodología}

\section{Objetivo}

Identificar elementos de riesgo, factores protectores y conductas resilientes, en un inmigrante colombiano, establecido en la zona central de Chile.

\section{Tipo de Estudio}

Estudio de caso único, analizado desde una perspectiva hermenéutica - comprensiva.

\section{Sujeto}

W. es un hombre de 32 años, que hace 7 años está viviendo en Chile. Actualmente trabaja y ha formado familia en el país. Sus labores principales están en el ámbito de la educación y el deporte.

\section{Técnica}

El levantamiento de la información se hizo a través de la realización de entrevistas en profundidad. Se trianguló la información a través de una entrevista con un amigo de W. que lo conoce hace varios años.

\section{Análisis}

Se hará un análisis comprensivo abierto, levantando categorías y construyendo núcleos de significado en torno a los conceptos de Riesgo, Protección y Resiliencia.

\section{Resguardos éticos}

Antes de la realización de las entrevistas, se firmó consentimiento informado, para el resguardo de la confidencialidad y una correcta utilización de los datos.

\section{Resultados}

Luego de las entrevistas llevadas a cabo durante el mes de octubre de 2015, presentamos los resultados de dichos encuentros.

Los datos se ordenan partiendo por los elementos de riesgo encontrados en su biografía, para luego identificar los factores protectores existente en su vida y se finaliza con la identificación de conductas resilientes presentes en el sujeto.

Para orientar mejor al lector, las verbalizaciones originales del sujeto, están escritas con letra cursiva y entre comillas.

\section{Condiciones de riesgo}

W. proviene de una familia de la ciudad de Cartagena de Indias, la que estuvo marcada por una temprana separación de los padres: "Mi papá,esaesunalargahistoria.Yololleguéaconocercuandoteníadoceaños,porquecuandoyo 


\section{Ciencia}

teníadosañossefuedelafamilia, porlotantono lo conocí". Esta situación marco la biografía de W. afectando su niñez: "No es lo mismo convivir consus padresqueconun padrastro,yaquesus hijoslosvaatratarmejorqueeldenosotros. Por lotanto,poreso másquetodomealejémás de lafamilia poresesentido", vivenciando situaciones de maltrato en su infancia: "Sí pegaba, en el sentidodequecuandoquisiera.Pegabaynospegabacomo...nocastigabaenbuenamanera.Por lo tanto mi relación con él nunca fue la mejor, poresome manteníafuera delacasa, fuerade la casa"

Nunca la relación con su padrastro fue buena y permanentemente entraban en conflicto: "él me decíaamíqueibaaserotrapersona,queibaaser undrogadicto, queibaa serunladrón,queibaa ser un mafioso".

Frente a estas situaciones la madre no tenía un rol protector hacia él y por el contrario dejaba que estas estuvieran presentes: "mi mamá era sumisa,erasumisa,alosdetalles...Porlotanto, no discutíamuchoconélenesesentido.Mecastigaba,yosabesqué,meibapa'dondemiabuelayallá me quedaba", buscando refugio fuera de casa.

En esta misma dirección agrega que: "bueno mimamáoseaapesardequenomedefendióenel momentoexactocuandomipadrastromecorreg... mecastigaba...yosentíaquenomequería...empecéahacerlascosasqueyoqueríahacer,perosin consultarleaél,ehtrasnochada,jugarjuegosde azar,sabemosquenoestápermitido,ycosasporel estilo, en ese sentido".

Dadas estas circunstancias en su infancia, W. debió tempranamente trabajar como cargador: "Yoempecéatrabajardesdelosdiezaños...yyo memontabaunbultodecincuentakilos, hastade cien kilos", con ello W. lograba reunir algún dinero para su subsistencia.

También el ambiente que rodeaba a la familia, estaba cargado de riesgos, siendo uno de ellos el consumo de drogas: "yo viví en esa circunstancia,enelsentidodequeteníamuchosfamiliares, amigos, que se perdían en eso".

Otra queja importante que aparece en el relato de W. es la falta de hábitos formados en la familia y la carencia de cariño: "desafortunadamenteamínomecrearonmuchoshábitos,yo vengodecrearloshábitoscuandoyaestoyviejo ya...Cuestaporquenotienesalgoestructurado en la vida...Creo que faltó mucho cariño en la casa,enelsentidoqueestuviesenmispadresunidos,quenosehubiesendivorciado,hubiesesido otralahistoria...cariñomaternalesotracosa... sífaltómucho... de papámuchomás todavía, mucho más".

Cuando ya estaba más adulto, W. tiene una gran dificultad al hacer negocios y perder mucho dinero: "sí, una vez caí, esa vez tenía que hacer unnegocio,hartosmillones,ylavozmedecíano lohagas, nolohagas, nolohagas.Peroeracomo ambiciosoquelohice,yperdí...Medestrocé,me 
destrocéenelsentidodequeanímicamenteme bajó,mebajé,mebajé...Paséunasemana,nodeprimido, pero sí triste".

Fue en esas circunstancias que W. sufrió el ataque y la descalificación de algunas personas: "quemeibaacostarlevantarme,quenoibaaser capazdesaliradelante,yqueotraspersonasme ibana señalar...sí, más de una vez me lo restregaron".

Al llegar a Chile su vida tampoco fue del todo plena y de alguna forma sintió los obstáculos que le ponían las personas: "al principio cuando yo lleguéaquíaChile,cuesta,enelsentidodequetú tienesqueromperunesquema,tienesqueromper unabarreraalasociedad deChile...porlotanto hay una barrera invisible". Barrera que se traducía en costumbres y valores diferentes, desde las comidas distintas, hasta la forma de ver la vida cotidiana:"Es decirnuncayo penséqueibaatenerunafamiliachilena, sabemosquesonculturas diferentes,soncostumbres, hábitos,ytocacómo compaginar un poco".

\section{Elementos protectores}

Dentro de las fuentes que proveían protección en W. encontramos en primer lugar a la figura de la abuela, fuente de comprensión y cariño: "Eh, cariñosa, mi abuela era mi madre.Sí, mi abuela eratodo,oseamiabuelameconsentía...vivimos unabuenainfanciaenelsentidoconellos,... de hechomihermanaqueestáaquíenChile,tenemosmuybuenosrecuerdosconella,losmejorMi
abuela...Ellaeslacentralahí,esla principal,ella es el pilar, es el pilar".

También aprende de la abuela una forma paciente de enfrentar los problemas: "Yo recuerdo quemiabuela, unadelascosasqueleaprendíy quelesigoaprendiendo,queesmuypaciente.Te puedenmartillar,tepuedentirarpiedras, peroella ahípaciente, paciente, paciente... Ella se hacía respetar". Señala además que la fuente principal de cariño en su infancia fue ella: "yo el cariño que recibí lo recibí de mi abuela y en la calle".

Justamente es en el ambiente extra familiar, donde W. también encuentra personas que lo apoyan y reconocen: "Éramos siete amigo muy unidos, decíamos... oyesabesqué,reunamosplatadeenerohastanoviembreyescogemosunpaís ynosvamos... Recorrimosel país,empezamosa cantar,ahídirigimosmuchascosas, muchaspersonasqueestabanenalcoholendrogas, losapoyamos, fuimosala cárcel;y deahínos vinimosa Chile.Actualmenteyohagoeso, hagotrabajoen lacárcel,enlagobernación,yen pequeñosgruposquenecesitenesto... Oseameacogieron,me dieroncariño,medieronternura,queesomucho lonecesitaunextranjero,enesesentidoanduvo bien".

Familias amigas también lo han apoyado para sus estudios y en el aspecto económico: "Por lotantomisamigoseranprofesores,licenciados, diputados, senadores, esossonlosamigosmíos, eran mi círculo. Por lo tanto, nunca yo fumé, ni tomé,nihicecosasquenodebíahacer...Jorge... 


\section{Ciencia}

yolorecuerdocon muchocariño, nosolamente unapersonaconfiable,unapersonaquemedecía enelmomentoexacto,cómoteníaquehacerlas cosasparaquenomeequivocara. Ellos(lafamiliadeJorge)meayudaronamí,... pagándomeel arriendo del departamento".

Otra fuente de protección es la religión y la figura de Dios, al respecto señala: "siempre Dios ha estadoconmigo,entodomomento,enlasbuenas yenlasmalas.Peroséquiénesmepuedenguiaro quienesmepuedendirigirdurantelavida, quienessonlosmásadultos,enelsentidodequeellos medanconsejos... YocreoquesiempreDiosha estadoconmigo,yosiemprehedichoquecuando unopuede,oquiere, puede... Yocreoquelareligiónesmuyimportanteparaelserhumano,es unabrújulaqueteda paradondetienesqueir... Dios me abrazó".

Los valores juegan un papel importante en la vida de W. y señala guiarse por ellos: "Nosotrossinorespetamosalosdemás, norespetamos aunapersonaqueesmayoranosotros, nosotros no somos nada. Tú respetas al mayor, el mayor teva respetarati... con esas clavesyollegaba a cualquierladoymetratabanbien". Agregaque algunas personas lo han guiado en torno a valores: "cuandotedanprincipiosycuandotedan ideales".

Los intereses personales y su esfuerzo también han acompañado la vida de W. y señala: "En elcolegionuncaperdíaaño,eh,estudiéenuncolegioprivado,mepagabantodo,porlomismo...me gustaba leer...escribir música, cantar".

Lo anterior redunda en que $\mathrm{W}$. desarrolle una auto estima positiva que lo hace desenvolverse bien en diferentes ambientes: "pasa que es como un don, es un don, porque mi autoestima, mi simpatíalehacecaerbienatodoelmundo...Yo esapersonalidadsefuedesarrollandomomento amomento,añoporaño,conociendoapersonas, conociendoapersonasquemedabanconsejos'tú tienesquepararteenestaforma,asíyasí'nodejes que violen tus derechos".

Apoyado en esa auto estima positiva, W. se ha involucrado en proyectos, desarrollando un rol activo en lo social: "Le propuse un proyecto para trabajarconjóvenesqueestabanenpeligrodedrogasyalcohol,meaceptóelproyectoymequedé... Empezamosatrabajarconjóvenesdelacomuna, yotrabajabaconlapartededeporte,ytrabajando enlapartededeporteyateconocemuchagente, porqueestásayudandoentodoslosclubesdeportivos".

Otro pilar importante y fuente de protección es la familia, principalmente la familia que ha formadoen Chile:"actualmenteconseguímipareja, tengounhijo,unhijochicolombiano,yahoratraje amihermana, quetieneunañoaquíen Chile, mi hermanatrabajaenunrestauranteymisobrinoestudiaenelcolegioSanRamón.Esossonpartedemi familiaahoraenChile...Actualmentemipresente estáguiándosebien,tieneunbuencimiento,ycon esebuencimientopodemosesperarelfuturo.Bueno enelpersonal,haestadobien,tengounhijodedos 
añosyochomeses...conmihermanasomosmugreyuña,nosotrosvamospatodaspartes,nosotros cuandoqueríamosillorar,llorábamoslosdosjuntos, sí,losdossomosmuyunidosenesesentido...elvalor delafamilia, que somos unidos, eso es lo que másrescato.Eseesunvalormuyfundamental porquecuandounomenospiensa,faltaalgo,peroellos estánahíapoyándote...quiénmeapoya,yocreoque meapoyamihermana,mipareja,mihijoymeapoya el jefe. Sí, sí hay gente que me quiere".

También hay proyectos con su actual familia: "Quieroqueestudielamedicina...porquesabemosqueloshijossinoloseducas, enelsentidode loscuatropilaresfundamentalesenlavida,quees lofísico,lomentalyloespiritualylosocial,esos4 pilares si no están juntos el niño va a decaer".

Finalmente una actitud optimista fortalece el quehacer de W. y agrega: "Siempre soy optimista...eloptimismoloapoyoenelsentidodeque puedequepaseopuedequenopase,perosiempre voyconungrado defequevaapasar, quelovoy alograr, aunquefracasemelevantaréyseguiré adelante".

\section{Conductas resilientes}

Desde su niñez W. mostró características resilientes, enfrentando situaciones difíciles y resolviendoproblemas:"yasabíacómodefenderme soloenlavida...Desdepequeño.Yomesostenía vendiendocosas.Entoncesesoamímesolventaba paratenerplataenmisbolsillosycomprarloque yoquería... latuveenmismanos, todo, peronun-
ca,yestonoespa'mi(ladroga).Yoyateníaclaroy específicopa'dondeibalabrújulamía,desdepequeño, ya sabía lo que tenía que hacer".

En etapas tempranas de su vida demostró enterezayfortaleza:"yoponíael pechonomas...pa' delante,noleprestabaatenciónanada,yosabía yapa'dondequeríahaceroquequeríahaceren elmomento... porlotantonomelamenté, alo contrario,volvímásfuerte...perositúeresfuerte, puedessalirmásadelantetodavía, mástodavía puedesllegarasermásgrande.Yahíestabayo... tenemosquelevantarnos, tenemosquelevantarnos, aunqueestélloviendofuerte,vamospa'delante... lavida te enseña cómo pararteo como estarfirmeenelmomentopropicio,es decirque sia tite molestan en la calle, tútienes que tener personalidadsuficienteparairapararesegrupo, decirlequeestánactuandomal,ysitieneneducacióncorrijanse.Solamentehablandoconeducación, las personas saben responder".

La misma experiencia de no vivir con su padre, la enfrenta de manera resiliente: "yo lo asimilocomoparabien,enelsentidodequenonecesité unpapá,perosíloconocícuandoteníalos 12años yélquisodarmesuapellido,mereconociócomosu hijoyyo"yano,oseanonecesitosuapellido...(le diceal padre) Sí, "yatengo 12 años, mi mamáes mipapáalmismotiempoyyosétrabajar,yyame sédefenderenlavida, porlotantomuchasgracias hastaaquínosvemos...yoqueríasaberquiénera usted, porlotantomedalo mismoloque haga, ahora en adelante logré saberquién era, yyala 


\section{Ciencia}

vidasigue... peroapesardetodoesoestoydondeestoy,apesardetodoeso...salíadelante,nome quedé en el pasado".

Esta fortaleza expresada, según W. tiene su origendentrodeél:"meheexpuestoamuchascosas...ysigoenlomío...graciasaDiostengobienclaro.Yséquetambiénlafuerzanoestáenelmúsculo,estáenelcerebro,saberhacerlascosasantesde hacerlas,enelsentidoquepuedeshacercualquier cosa,peroavecescometeserroresquetevasalamentar, poresounotienequepensarmuybien".

Incluso con el humor, combate algunos ataquesydescalificaciones: "noletomoimportancia alapersonaquetequierehacerdaño.Túsiempre tienesquetenerlamentefija,elpechoelevadoy seguiradelante,siempreenlavida...conbuenhumor,hagolonecesarioparaqueelfracasonoexista, hagolonecesarioparaqueeltriunfosíexista".

W. une la idea de ser algo o alguien en la vida con ejercer un rol social, y es así como él expresa estaárea:"yotengoqueseralgooalguienimportante,nosolamenteparamímismo,sinoparalas personasquemerodeanypa'lasociedad,aportar algoalasociedad...enelsentidodeladelincuencia, aldelincuenteselerechaza porsertal como es, perosabemosquesial delincuentedanbuenoshábitos,selepuedecambiareseconcepto,se lepuedecambiar.Dehechoyoheidoalacárcel ahacerobrassocialesconellos...unavezpenséy dije,algúndíayoayudaréapersonascomoellos,y actualmente lo estoy haciendo".
Unido a lo anterior desde pequeño $\mathrm{W}$. quiere torcerle la mano a ese destino de pobreza: "Esa motivaciónnace,cuandotedanprincipiosycuandotedanideales.Queparanosotros, parapoder salirdepobres, hayqueestudiar.Porqué,porque los conocimientos y los libros, te hacen libre... deahímelevantéyseguíluchando... aunquete caigas levántate, porque no estás solo". Incluso en contra de las predicciones de su padrastro en torno a que sería igual que los del barrio...drogadicto...delincuente, W. señala que le cambió esa convicción.

Algunos proyectos actuales fortalecen aún más la vida de W.: "sí, en estos momentos estamosoptandopa'futurotenersupropiacasa,tener casa". Del mismo modo hoy día tiene planes de hacer una carrera política, para ejercer de mejor manera un rol social.

W. finaliza su relato agregando los elementos de determinación y aprendizaje en su conducta:"sabemosqueenlavidanosotroscometemos muchoerroresytenemosquedefendernosdeesos errores,seguiradelante, noquedarseenelvacío, hayquesalir...esdecir,sitúsabesquetienesque lograralgolovasalograr,aunquetetiren piedras, aunqueseteacabeelmundo, perotútienesuna meta, unhorizonte.Sinosotrosnostrazamosalgo desde el principio, lo podemos lograr".

\section{Conclusiones}

En coherencia con los estudios anteriores, las situaciones de ruptura familiar temprana, apa- 


\section{Ciencia}

recen como elementos de riesgo que marcan el desarrollo posterior de W. Del mismo modo, las experiencias de maltrato físico en la infancia y la nula protección de la madre, constituyen otro elemento de riesgo.

El trabajo infantil aparece como otra situación de riesgo, debiendo asumir labores pesadas y permaneciendo en la calle por largos períodos. Referido a esta permanencia en la calle, adquiere relevancia el ambiente del barrio, que está cargado de situaciones de consumo y tráfico de drogas.

Para el caso analizado, el hacer malos negocios en la adultez, lo ponen en una situación crítica de dinero y sufre el rechazo de otros.

Al llegar a Chile, también está en una situación de riesgo, ya que debe enfrentarse con otra cultura con diferentes costumbres, a la vez de haber sufrido algunos ataques personales por su color de piel y nacionalidad.

Por su parte en torno a factores protectores, aparece la abuela, como primera y central figura de protección, que reemplaza incluso a la figura materna, la cual es percibida más distante y sumisa frente a su padrastro. La abuela también, se convierte en un modelo para el afrontamiento de problemas, enseñándole una forma paciente de actuar.

Los amigos son una fuente de apoyo y protección. A ellos acude cuando necesita consejos o bien ante las carencias económicas.

Otra fuente central de protección y orientación, se encuentra en la religión y sobre todo en la figura de Dios. Confesará que no realiza nada re- levante sin antes pensar en Dios y ponerse en sus manos. Este punto, diversos estudios lo señalan como fuente de protección.

Ligado a lo anterior, el desarrollo de valores y la educación, le ofrecen a la persona, una orientación ante los desafíos y problemas. La educación pasa a ser uno de los pilares de la construcción de sus conductas. El valor de aprender de los adultos, en particular, ocupa un lugar central a la hora de enfrentar las dificultades.

Como se señalara en el apartado teórico, una autoestima positiva y una actitud de optimismo ayudan a fortalecer a las personas, que en este caso particular nuestro sujeto reconoce tener esta disposición para la solución de sus problemas.

A la vez la familia que ha formado en Chile, se ha convertido en fuente de apoyo y motivación. Es en torno a ella que aparecen algunos proyectos $\mathrm{y}$ tareas a futuro.

Una de las primeras conductas resilientes que aparece en esta persona, es el enfrentar su situación de pobreza económica, desarrollando trabajos para solventar sus gastos. Llama la atención como esta situación objetiva de riesgo, la transforma en una oportunidad de salir adelante.

Una actitud positiva frente a los ataques y las descalificaciones, lo acompañan para resolver dichas situaciones. Siempre hablé de salir adelante, de enfrentar los obstáculos, de apoyarse en la educación. La misma experiencia de crecer sin su padre, la transforma y hace frente a sus carencias materiales y afectivas, asegurando no necesitar 


\section{Ciencia}

dicha figura o reemplazándola por otras figuras significativas.

Aspectos como la habilidad intelectual, la fe, la simpatía y el humor, son descritos como elementos en los que se apoya la conducta de esta persona para afrontar sus dificultades, lo que resulta muy coherente con características descritas en la literatura acerca de resiliencia.

Finalmente, una conducta claramente constructiva y resiliente, la constituye el rol social que ejerce en algunas acciones: trabajar en la cárcel, asesorar a jóvenes drogadictos, hacer labores de

\section{Referencias}

Bajraj, R., Villa, M. y Rodríguez, J. (2000). Población y desarrollo en América Latina y el Caribe: un desafío para las políticas públicas. Serie Políticas y desarrollo, 7, 1-46.

Organización para la Cooperación y el Desarrollo Económico (2014). Informe Anual. París: Autor.

Rodríguez, J. y Busso, G. (2009). Migración internaydesarrolloenAméricaLatinaentre 1980 y 2005. Santiago: CEPAL. orientación. El aprendizaje de los errores también, está presente en su relato, en el sentido de construir algo positivo desde los obstáculos y con determinación para salir adelante.

Podemos identificar claramente entonces los elementos de riesgo y protección en su biografía y como algunos de ellos, son acentuados por la situación de migración permitiendo en este caso, ocupar sus recursos internos para combatir sus dificultades y proyectarse hacia un futuro constructivo.

Saavedra, E. (2011). Investigación en resiliencia: algunosestudioscualitativosycuantitativos. Alemania: E. Académica Española.

Stefoni, C. (2012). Migración, remesas y desarro1lo, Polis 30, 2-19. DOI 104000/polis.2389.

Villa, M. y Martínez, X. X. (2001). Resumen y aspectos destacados del Simposio sobre Migración Internacional en las Américas. Serie Políticas y desarrollo, 14, 1-82. 